\title{
A novel approach to assess collaborative learning processes and group performance through the knowledge convergence
}

\author{
Lanqin Zheng • Nian-Shing Chen • \\ Ronghuai Huang $\cdot$ Kaicheng Yang
}

Received: 28 April 2014/Revised: 29 June 2014/Accepted: 30 June 2014 /

Published online: 22 July 2014

(C) Beijing Normal University 2014

\begin{abstract}
The challenge of assessment in collaborative learning is well known. The major issue is whether the assessment should focus on the individual level or the group level. Traditional assessment approaches only concern about individualized assessment, examination, or coding individual transcripts into speech acts. How to support teachers to monitor and assess collaborative learning processes at the group level has not been systematically addressed. This paper aims to design a new approach for assessing collaborative learning processes and group performance through the lens of knowledge convergence. We use the innovative knowledge map approach to analyze the degree of process and outcome convergence so as to provide insight into the qualities of collaborative learning processes. A total of 94 undergraduate students participated in this study. The empirical results indicate that the qualities of process can be quantified by the number of activated common knowledge and the degree of process convergence. The degree of outcome convergence can be used as an effective indicator for assessing group performance. Implications for instructors to facilitate knowledge convergence in collaborative learning are also discussed. The major contribution of this study is the design of a
\end{abstract}

\footnotetext{
L. Zheng

http://ksei.bnu.edu.cn/zhenglq/English/Eindex.html

e-mail: bnuzhenglq@bnu.edu.cn

N.-S. Chen $(\square)$

http://www.nschen.net

e-mail: nianshing@gmail.com

R. Huang

http://ksei.bnu.edu.cn/en/staff/staff_huangrh.htm

e-mail: huangrh@bnu.edu.cn

K. Yang

http://ksei.bnu.edu.cn/Yangkc/index.htm

e-mail: yangkc_beijing@foxmail.com
} 
novel approach to assess a collaborative learning process and outcome by analyzing the degree of knowledge convergence.

Keywords Collaborative learning - Knowledge convergence · Assessment · Knowledge map · Group performance

\section{Introduction}

Collaborative learning has become very important at all levels of education. However, assessment of collaborative learning is mainly summative and comprises individual learning achievements by pre-test and post-test or essays (Gress et al. 2010; Strijbos 2011). Researchers have pointed out that summative assessment which conducted after the collaboration is isolated from the learning process (Strijbos 2011). In fact, what is more important is the quality of the collaborative learning process that can provide insights into comprehensive pictures of a collaborative learning progress. Although previous studies have explored ways to assess the processes by analyzing individual student portfolios (Macdonald 2003; van Aalst and Chan 2007) or coding discourse transcripts (Fahy et al. 2001; Kapur et al. 2011; Meier et al. 2007; Nandi et al. 2012), very few studies were conducted to assess collaborative learning processes and group performance from the perspective of knowledge convergence.

Fischer and Mandl (2005), however, noted that further study is needed on knowledge convergence in collaborative learning. Recently, knowledge convergence has attracted substantial attention in collaborative learning research (Kapur et al. 2011; Spemann and Fischer 2011; Zottmann et al. 2013). It has been generally assumed that knowledge convergence means all group members' understanding become more similar after collaboration (Ickes and Gonzalez 1996; Jeong and Chi 2007; Klimoski and Mohammed 1994; Weinberger et al. 2007). Jeong and Chi (2007) defined knowledge convergence as an increase in common knowledge, where common knowledge refers to similar knowledge. Weinberger et al. (2007) further defined knowledge convergence as knowledge equivalence and as shared knowledge. Knowledge equivalence refers to learners becoming more similar to their team mates with respect to their knowledge, while shared knowledge means that learners have the same concepts among their group members. In a word, knowledge convergence puts emphasis on increasing similarity of group members with regard to their knowledge.

Most theoretical approaches assume a mutual influence among group members via social interactions in a collaborative learning setting (Strijbos and Fischer 2007). Knowledge convergence can serve as a vehicle for testing theoretical assumptions of learners' mutual influence, which is regarded as the crucial aspect of collaborative leaning (Roschelle 1996). As a group-level phenomenon, knowledge convergence is viewed as evidence that collaborative learning has occurred. Fischer and Mandl (2005) found that learners who converged in knowledge benefited more from collaborative learning than learners who do not. Group members are also 
characterized by convergence of knowledge which is primarily conceptualized as the process of co-construction of shared understanding in collaborative learning (Roschelle and Teasley 1995; Stahl et al. 2006). While shared understanding is mainly achieved by social interactions in collaborative learning. Recently, researchers have pointed out that the current approaches in collaborative learning have moved on from simple coding discourse data of the social interaction process to quantify certain qualities of collaborative learning processes (Spemann and Fischer 2011).

Previous studies assessed the qualities of collaborative learning processes based on certain coding schemes to count speech acts (Weinberger and Fischer 2006; Zhang et al. 2009). In fact, coding discussion transcripts into speech acts is very difficult because the real meanings of human's speech acts are implicit. Also, the identification of speech acts is very subjective. Moreover, some knowledge construction processes will be ignored if only analyzing the explicit speech acts. Therefore, this study aims to design a novel approach for assessing collaborative learning processes and group performance through the lens of knowledge convergence. This new approach analyzes knowledge convergence based on information flows during a collaborative learning process. The information flows can be mapped onto knowledge maps by which the convergent knowledge can be automatically calculated. The research questions of this study are described as follows:

RQ1: How to assess the quality of a collaborative learning process through the knowledge convergence?

RQ2: Can the degree of knowledge convergence predict group performance?

RQ3: What is the predicting power of the developed knowledge convergence indicators compared to other commonly used predictors?

\section{Literature review}

\section{Related work}

Various collaborative learning assessment approaches have been reported in the literature. For example, social network analysis method that investigating relationships among group members (Aviv et al. 2003; De Laat et al. 2007) content analysis method that analyzing the knowledge construction (Weinberger and Fischer 2006) or depth of understanding (Zhang et al. 2009), conversation analysis that identifying action sequences or turn-taking (Zemel et al. 2005), interviews or questionnaires for evaluating the perceptions of students during collaborative learning (Jarmon et al. 2009; Li et al. 2012; Prokofieva 2013). However, the assessment of collaborative learning processes as well as learning outcomes from the perspective of knowledge convergence remains lacking.

Knowledge convergence has been conceptualized in various ways. According to Roschelle (1996), convergence means that two or more learners' activities have an 
effect on those of their team members, which, in turn, have an impact on their own activities. Ickes and Gonzalez (1996) argued that knowledge convergence refers to making the cognitive responses of group members more uniform. Weinberger et al. (2007) conceptualized knowledge convergence as knowledge equivalence and shared knowledge. This implies a shift focus on the process and outcome of knowledge convergence by emphasizing knowledge sharing through discussion. As a consequence, they argued that knowledge convergence outcomes should center on knowledge equivalence, i.e., learners become more similar to their team mates after collaboration. Jeong and Chi (2007) operationalized knowledge convergence as an increase in the knowledge that all collaborators possess.

We argue that knowledge convergence mainly focuses on how learners become more similar in understanding with regard to their knowledge during a collaborative learning process and how common knowledge is constructed during and after collaboration. Previous study has noted that knowledge convergence is empirically linked to the effectiveness of collaborative learning (Fischer and Mandl 2005). In order to assess the effectiveness of collaborative learning, a better measure of knowledge convergence is necessary. Researchers have explored how to measure the extent of knowledge convergence in different ways. For example, Fischer and Mandl (2005) measured convergence in resource usage based on Euclidean distances between individual frequencies of the six categories of resource usage. Weinberger et al. (2007) assessed knowledge convergence by measuring knowledge equivalence and shared knowledge prior to, during, and after collaborative learning. Knowledge equivalence was measured based on the coefficient of variation of individual test scores, while shared knowledge was calculated using the score of pair-wise comparisons of knowledge tests divided by the mean value of the group. As Weinberger et al. (2007) mentioned, this kind of measurement was susceptible to ambiguity of individuals' contributions. Jeong and Chi (2007) claimed that the amount of convergence was measured by the increase in common knowledge, which can be calculated by subtracting the amount of common knowledge at the pre-test from the amount of common knowledge at the post-test. While Kapur et al. (2008) computed the knowledge convergence by the mean distance of the Markov walk, which can be calculated using the following formula (1)

$$
C=\frac{n_{1}-n_{-1}}{n_{1}+n_{-1}},
$$

where $n_{1}$ denotes interactions that move toward a goal state and $n_{-1}$ denotes interactions that move away from a goal state.

In addition, Clariana et al. (2011) tried to use degree centrality of a graph to measure the level of group knowledge convergence. Degree centrality of a graph can be calculated using the following formula (2):

$$
C_{D}(G)=\frac{\sum_{i=1}^{v}\left[C_{D}\left(v^{*}\right)-C_{D}\left(v_{i}\right)\right]}{\max \sum_{i=1}^{v}\left[C_{D}\left(v^{*}\right)-C_{D}\left(v_{i}\right)\right]},
$$


where $\mathrm{C}_{\mathrm{D}}\left(v_{\mathrm{i}}\right)$ denotes the degree centrality of node $v_{\mathrm{i}}$ and $\mathrm{C}_{\mathrm{D}}\left(v^{*}\right)$ denotes the highest degree centrality.

Therefore, most of the measurements of knowledge convergence mainly depend on the pre-test and post-test of individual learning outcomes, which cannot help in understanding the complex convergent processes at the group level. In addition, the measurement neither traces processes of knowledge convergence nor considers systematic variations on team knowledge. In summary, previous research on knowledge convergence suffers from a lack of systematic conceptualization and operationalization of the convergence construct.

The present study

In this study, we tried to develop an operational definition and quantitative measurement of knowledge convergence in a more precise manner. We conceptualize knowledge convergence from the perspective of how much common knowledge was activated during and after collaborative learning. We argue that knowledge convergence is the degree of activating common knowledge among teammates. Knowledge convergence can be viewed from the process of collaborative learning or from the final learning outcomes after collaboration. Therefore, we consider two main aspects of knowledge convergence, process convergence, and outcome convergence. Process convergence is defined as the common knowledge nodes which are continuously activating at any given period of time in a collaborative learning process. Outcome convergence is defined as the overall activated common knowledge nodes after collaboration. Here, common knowledge refers to the knowledge nodes that activated by all the members. The activation quantity of common knowledge nodes can be computed by information entropy generated through the interactions, which can be calculated according to the formula (3) (Zheng et al. 2012).

$$
A_{i}=\sum \frac{F * \log (d+2) * r}{\log (n *(D-d+2))}
$$

This notion of knowledge convergence as just described can derive two operational and computational formulas for calculating the degrees of process convergence and outcome convergence which will be described in the next section.

\section{Method}

\section{Participants}

There were 94 students enrolled in educational psychology classes participated in the study ( 69 female, 25 male). The average age of the participants was 20 years old. The participants were randomly divided into 31 groups of three or four. None of them had interacted with their classmates prior to the study. Thus, a total of 31 groups participated in the experiment. 
Collaborative learning tasks

The collaborative learning task focused on application-oriented knowledge. Note that all participants were assigned the same tasks. They applied the theory of knowledge transfer such as identical elements theory, generalization theory to solve the following three problems in collaboration with their team mates:

- What are the opinions and limitations of various theories of knowledge transfer? How can these theories be improved?

- Please illustrate some instructional strategies of promoting knowledge transfer when instructing the concept of a rhombus and the height of a triangle.

- How to apply the theory of knowledge transfer to explain the concept of the dihedral angle and the judgment of two perpendicular lines to the plane?

\section{Experimental procedure}

The experimental procedure consisted of three stages, that is, conducting the pretests, collaborative learning missions, and conducting the post-tests. In the first stage, a pre-test of content-specific knowledge was administered to all 94 participants. The total time of this stage was $20 \mathrm{~min}$. In the second stage, the groups collaborated face-to-face with the aid of mobile phone in different lab rooms. If they have any question, they could search related information via the Internet using mobile phone. Free Wifi was provided in each lab. The time for this stage was about $90 \mathrm{~min}$. In the final stage, a post-test was administered to assess group performance. The total time of this stage was also $20 \mathrm{~min}$. The collaborative learning task for each group as well as the items on pre-test and post-test was identical. No specific instructions were given, thus allowing collaborative learning to occur in naturalistic situations. The collaborative learning processes of each group were videoed so that the processes and outcomes of knowledge convergence could be analyzed. Two coders rated all items on the pre-test and post-test, and all of the reliability values (Cohen's kappa) were above .90 .

\section{Measures}

In this study, we used the knowledge map approach to measuring the degree of knowledge convergence. In a knowledge map, nodes represent knowledge, and edges represent mutual relationships. The categories of knowledge include symbols $(\mathrm{SM})$, concepts $(\mathrm{CN})$, principles and formulas $(\mathrm{PF})$, formats $(\mathrm{FM})$, processes and steps (PS), cognitive strategies (CS), and facts and cases (FC). The following three steps were conducted to analyze and measure the degree of knowledge convergence with the help of the developed analytic tool:

1.Draw an initial knowledge map according to the collaborative learning objects and tasks. In this study, the object of the collaborative learning is to understand the 


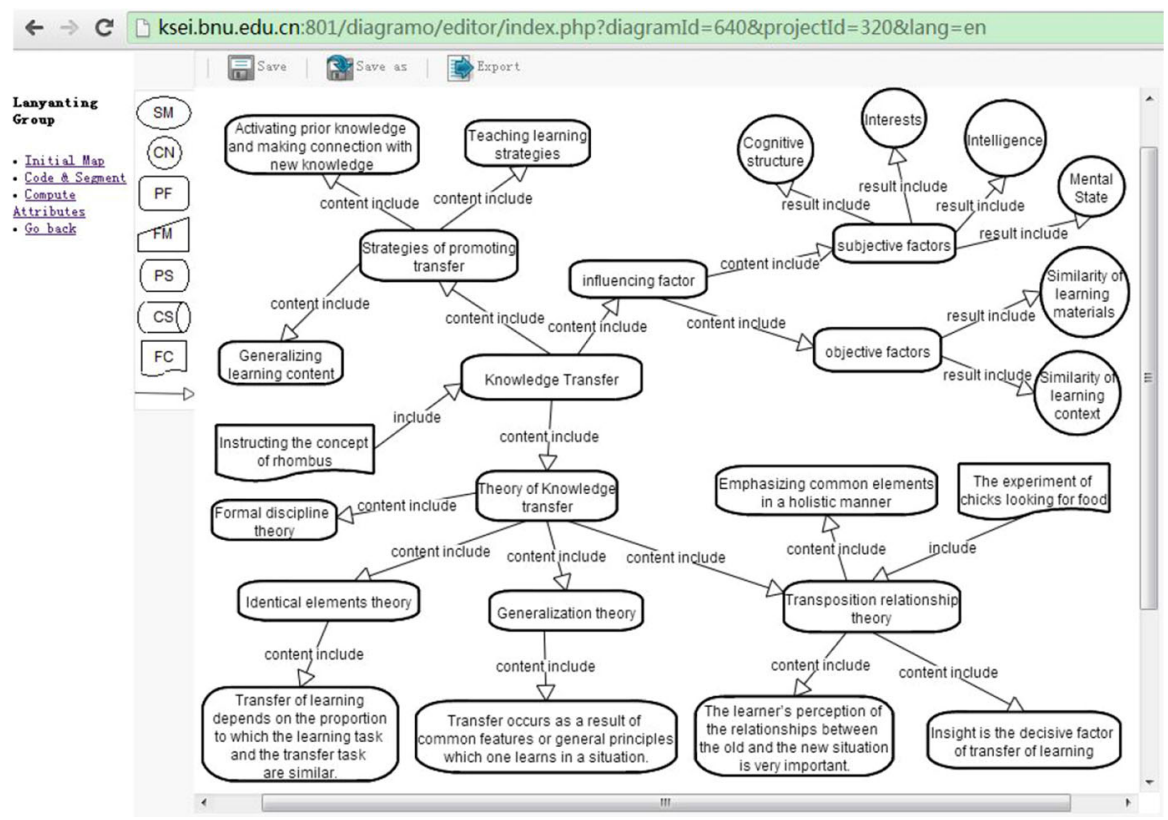

Fig. 1 Some portions of the initial knowledge map

theory of knowledge transfer and apply the theory to an instructional context. Figure 1 shows some portions of the initial knowledge map.

2. Code and segment information during the interaction process according to the coding format. Each information flow is coded into: $<$ time $><$ IPLi $><$ cognitive level $><$ information type $><$ representation format $><$ knowledge sub-map $>$. "Time" denotes the start time of each information flow, "IPLi" denotes information processing of learners, and " $\mathrm{i}$ " is used to distinguish different learners. "Cognitive level" includes recalling, understanding, and applying. "Information type" is categorized into objectives, context, knowledge semantics, management instructions, related information, and unrelated information. "Representation format" includes text $(\mathrm{T})$, sound $(\mathrm{S})$, graph $(\mathrm{G})$, photo $(\mathrm{P})$, table $(\mathrm{Tb})$, video $(\mathrm{V})$, animation (A), object (O), and body language (B). "Knowledge sub-map" is mapped into initial knowledge map, which can represents knowledge and their relationships. Two coders coded all of the data of thirty-one groups independently. Inter-rater reliability values regarding these analyses are above 0.91 , measured with the percent agreement index. Table 1 shows fragments of information of a group. They are coded and segmented into information sequences (see Fig. 2). These data were analyzed to assess learning process convergence as well as outcome convergence.

3. Compute the degrees of process and outcome convergence. In this study, an innovative measurement method was designed for calculating process convergence and outcome convergence. The degree of process convergence can be calculated using the formula (4): 
Table 1 Fragments of information during the interaction process

Time IPL $_{\mathrm{i}}$ Information during the interaction process

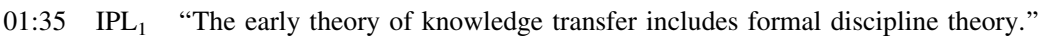

01:37 IPL $_{3} \quad$ "I think that the theory of knowledge transfer also includes identical elements theory."

02:40 $\mathrm{IPL}_{3} \quad$ "However, the transposition relationship theory is a very familiar one to me."

03:05 IPL $\mathrm{IL}_{1} \quad$ "Oh! The transposition relationship theory emphasizes that the learner's perception of the relationships between the old and the new situation is very important."

03:12 IPL IP $_{3} \quad$ "Yes, you are right. In addition, this theory highlights insight is the decisive factor of transfer of learning."

03:17 IPL 2 "Yes. I agree with this view."

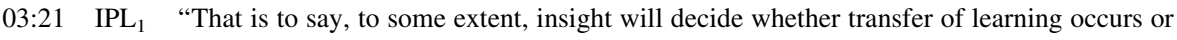
not."

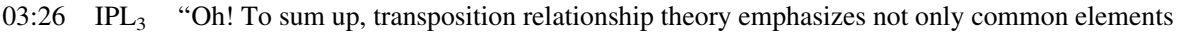
in a holistic manner but also the insight of learners."

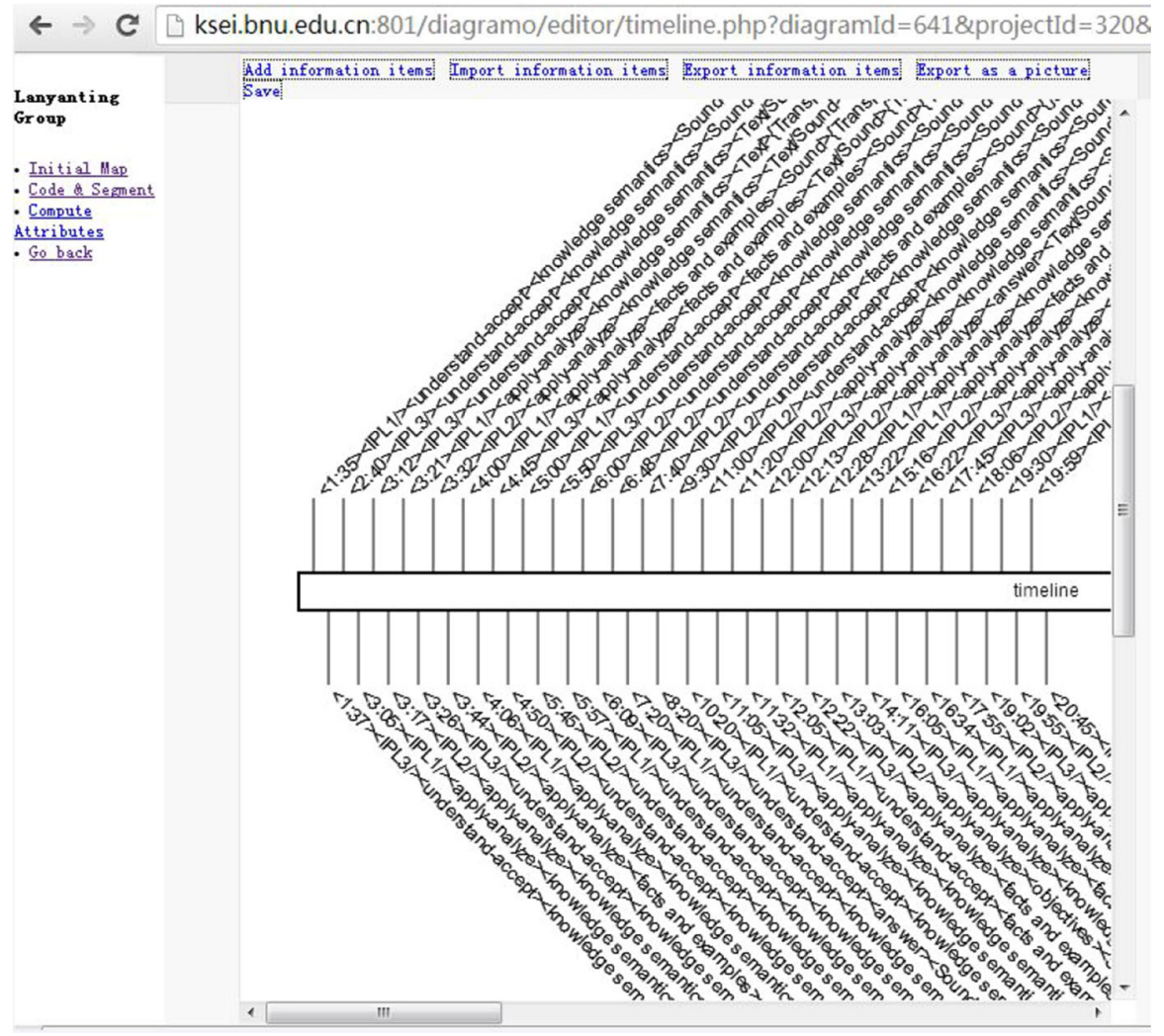

Fig. 2 Fragments of the information sequences 


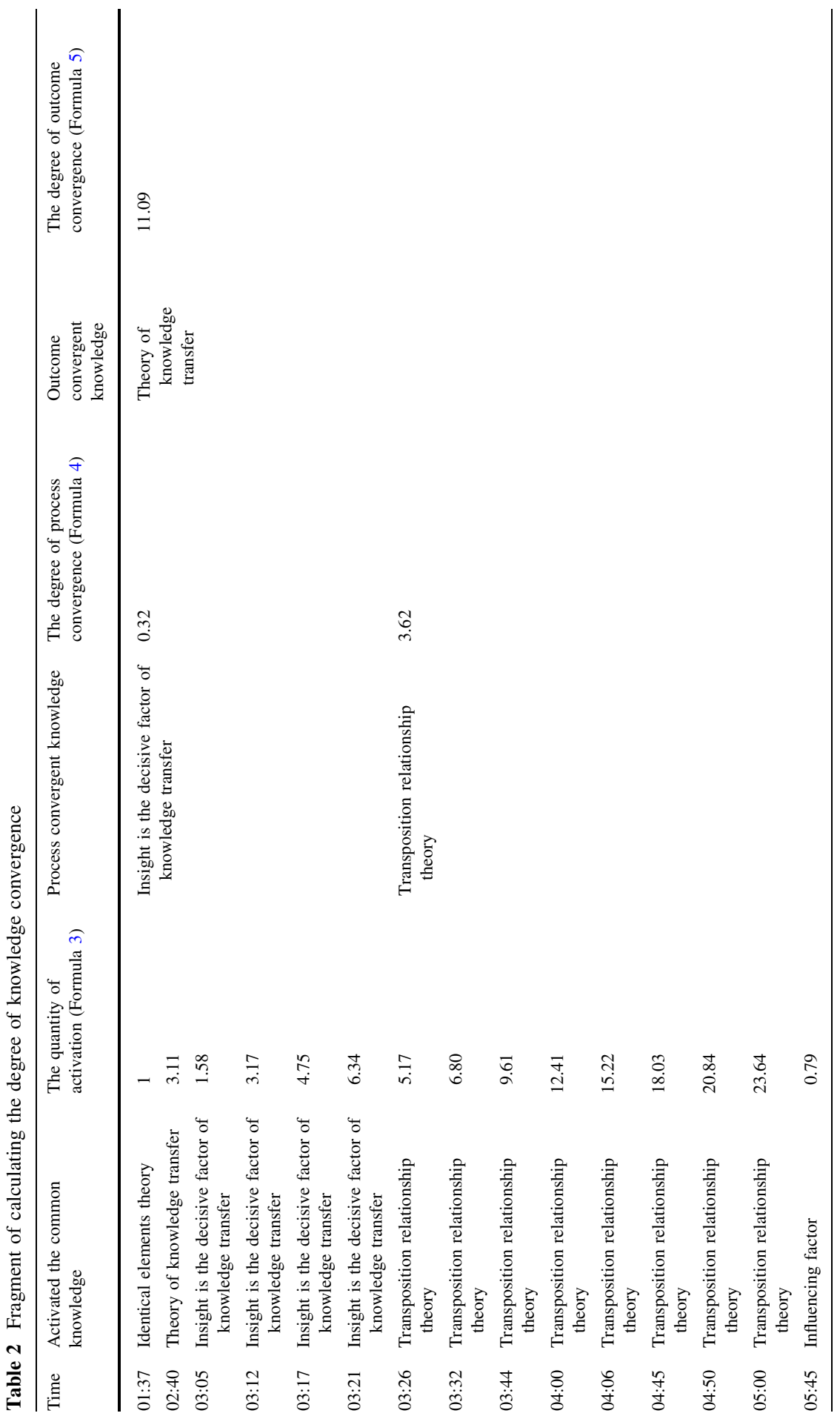




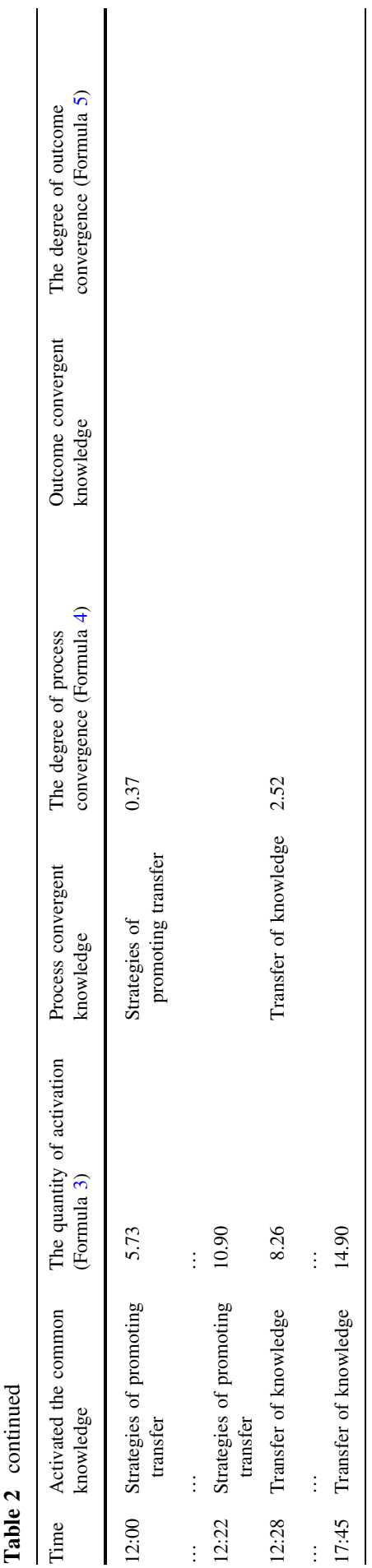




$$
C_{p}=\frac{\Delta A_{i}}{n} \times \Delta t,
$$

where $\Delta A_{i}$ denotes the increment of quantity of activation which can be calculated via the formula (3), $\mathrm{n}$ denotes the number of the common knowledge which was continuously activated at a given period of time,

$\frac{\Delta A_{i}}{n}$ denotes the average increment of activation quantity, and $\Delta t$ denotes the duration of collaboration. The degree of process convergence can be calculated via the analytical tool and exported into an Excel file, as shown in Table 2.

Outcome convergence aims to assess the degree of convergence after collaboration. The degree of outcome convergence can be calculated using the formula (5):

$$
C_{o}=\sum_{i=1}^{n} \frac{C_{p}}{\frac{\frac{1}{S_{i}+1}}{\mathrm{e}^{\mathrm{S}_{i}+1}-1}},
$$

where $\mathrm{C}_{\mathrm{p}}$ denotes the degree of process convergence that can be calculated using formula (4) and $S_{i}$ denotes the path length between the actual process convergent knowledge and the expected process convergent knowledge in the knowledge map. The actual process convergent knowledge can be identified by computing the activation quantity using the analytical tool. While the expected process convergent knowledge can be identified by teachers according to the collaborative learning objectives. The path length is defined as the sum of the edge lengths along the path.

$\frac{\frac{1}{s_{1}+1}}{e^{\frac{1}{i}++}-1}$ denotes the distance between the actual process convergent knowledge and the expected process convergent knowledge which is manually calculated, and $\mathrm{n}$ denotes the number of activated common knowledge .

Only the degree of process convergence is greater than one, the knowledge can be selected as the convergent knowledge when computing the degree of outcome convergence. For example, the convergent degree of "insight is the decisive factor of transfer of learning" and "strategies of promoting transfer" in Table 2 was less than one, so they were ignored because the degree of process convergence was too low to be included.

\section{Results}

How to assess the quality of a collaborative learning process through the knowledge convergence? (RQ1)

In order to assess the quality of a collaborative learning process, we first tried to visualize the whole interaction process using a knowledge map at any given time. The aforementioned analytical tool was adopted to generate the knowledge map. The information sequences were regarded as the input (see Fig. 2), and the corresponding knowledge map can be automatically generated as an output (see Fig. 3). The interaction process of each group can be clearly visualized to 


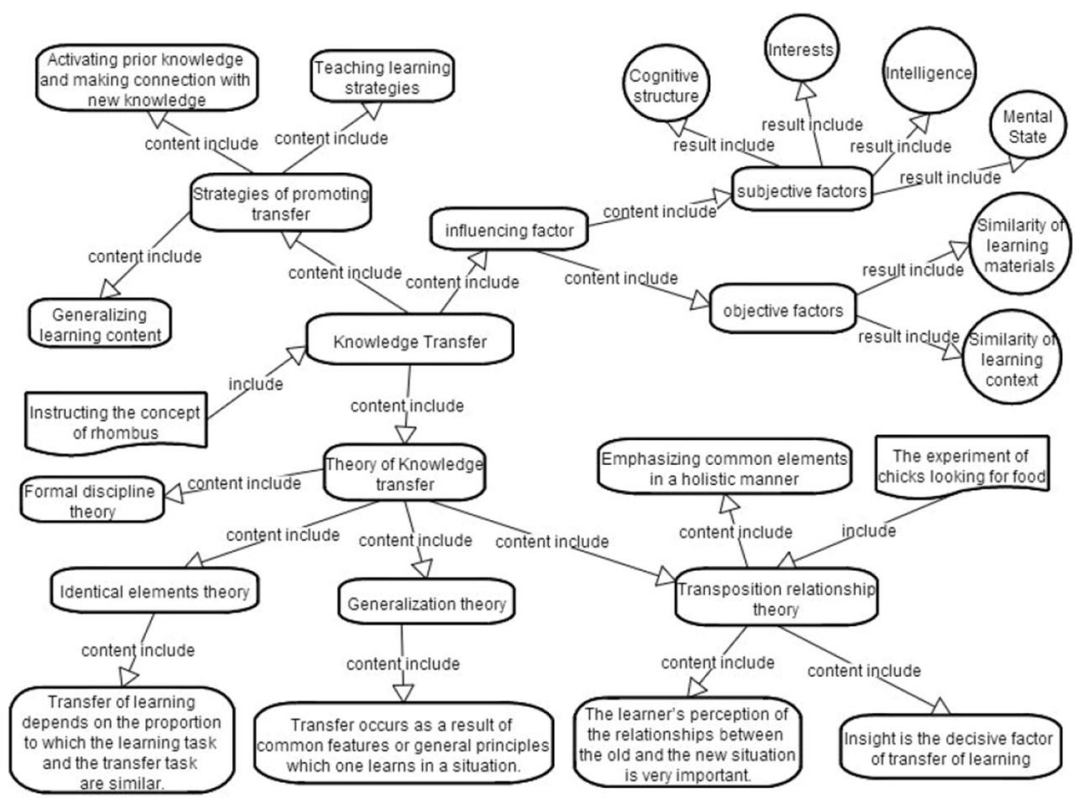

Fig. 3 A snapshot of knowledge activation in a collaborative learning process

understand the whole process of knowledge construction with the help of the analytic tool.

We then quantified the quality of a collaborative learning process by computing and visualizing the degree of process convergence using the developed analytic tool. Figure 4 shows the process of knowledge convergence over a certain period of time in one group. As shown in Fig. 4, nodes with yellow denote the process convergent knowledge, which are presented in the fourth column of Table 2. The number beside each of the process convergent knowledge denotes the degree of process convergence of that knowledge node as presented in the fifth column of Table 2. All the process convergent knowledge and the degree of process convergence can be clearly visualized and identified in Fig. 4. Teachers can easily compare whether the process convergent knowledge was consistent with the expected convergent knowledge by examining the knowledge map shown in Fig. 4. Teacher can also identify which knowledge has the best convergence by comparing the degree of the process convergence.

Additionally, previous studies have reported that the total quantity of activation which can be calculated via formula (3) is significantly related to group performance (Zheng et al. 2012). Therefore, we tried to analyze the relationships between the number of activated common knowledge and the total quantity of activation. The results also revealed that the number of activated common knowledge was related to the total quantity of activation $(r=.575, p=.001)$. This 
Table 3 Regression parameter values of different predictors

\begin{tabular}{llrrr}
\hline Predictors & Adjusted R & Beta & $t$ & $p$ \\
\hline Shared knowledge & .009 & .205 & 1.126 & .269 \\
An increase in common knowledge & .054 & .293 & 1.650 & .110 \\
The mean distance of the Markov walk & .057 & .298 & 1.681 & .104 \\
Degree centrality of a graph & .082 & -.335 & -1.915 & .065 \\
The degree of outcome convergence & 1.013 & .487 & 3.002 & 0.005 \\
\hline
\end{tabular}

indicated that more knowledge convergence led to better quality of a collaborative learning process. To sum up, the degree of process convergence and the number of activated common knowledge can be used to assess the quality of a collaborative learning process.

Can the degree of knowledge convergence predict group performance? (RQ2)

We examined whether the degree of outcome convergence can predict group performance. Group performance was operationalized as the result of pre-test and post-test, which can be calculated using the formula (6) (Zheng et al. 2012).

$$
\mathrm{X}=\frac{\mathrm{P} \times\left(\sum_{\mathrm{i}=1}^{\mathrm{N}} \mathrm{X}_{\text {iposttest }}-\sum_{\mathrm{i}=1}^{\mathrm{N}} \mathrm{X}_{\text {ipretest }}\right)}{\mathrm{N} \times \sqrt{\mathrm{CV}}}
$$

The result indicated that the degree of outcome convergence was significantly positively correlated with group performance $(r=.487, p=.005)$. Furthermore, a linear regression analysis was conducted to examine the predictive validity of knowledge convergence on group performance. The results showed that the degree of outcome convergence could explain $21.1 \%$ of the total variance $(\beta=.487$, $t=3.002, p=0.005)$.

What is the predicting power of the developed knowledge convergence indicators compared to other commonly used predictors? (RQ3)

We also compared the predictive power of the developed indicators with other commonly used predictors. In this study, we operationalized four other measures to represent typical measures: shared knowledge proposed by Weinberger et al. (2007), an increase in common knowledge proposed by Jeong and Chi (2007), the mean distance of the Markov walk proposed by Kapur et al. (2008), and degree centrality of a graph proposed by Clariana et al. (2011). The data were collected from the post-test and discourse transcripts of thirty-one groups in this study.

Table 3 shows some parameter values of five different predictors. According to results from regression analysis, shared knowledge, an increase in common knowledge, cannot predict group performance. The reason for this result is that these two indicators used simple measurement without considering the process of 


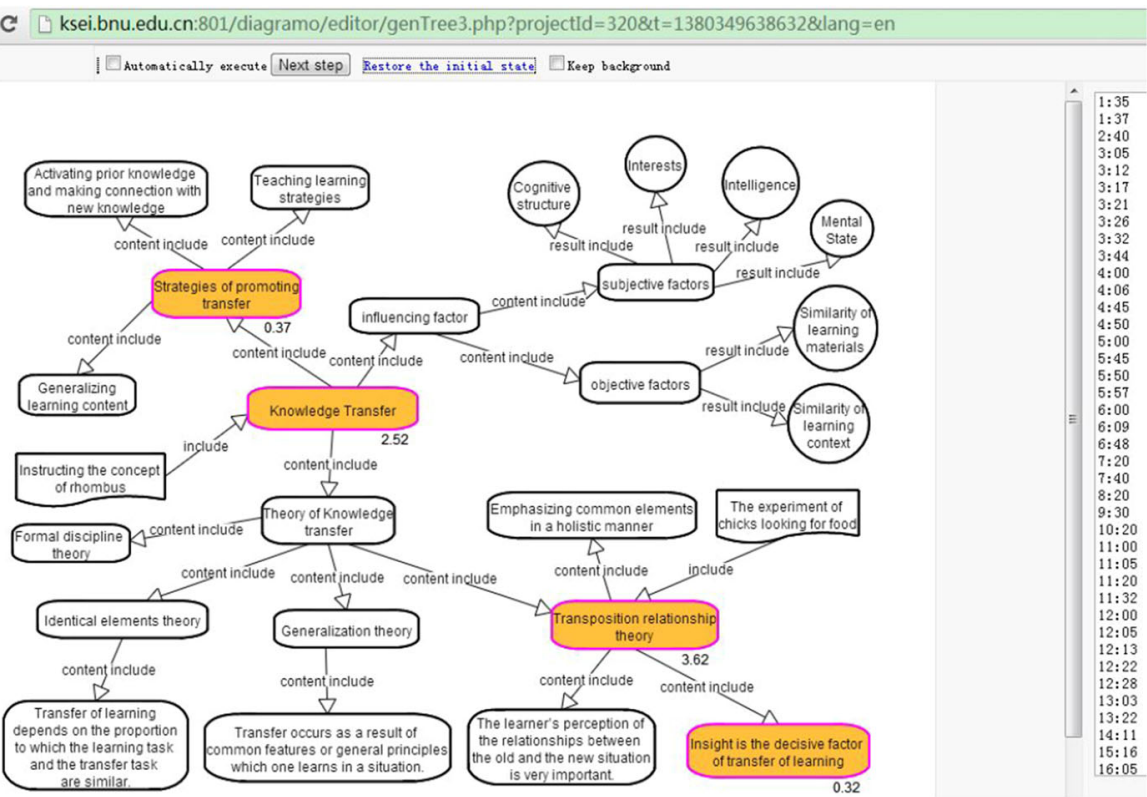

Fig. 4 The process convergent knowledge and the degree of process convergence

knowledge convergence. The mean distance of the Markov walk cannot predict group performance either. The reason for this is that the judgments of interactions moving toward or away from a goal are very subjective and ambiguous because of lacking an objective reference. Table 3 also indicates that degree centrality of a knowledge map cannot predict group performance. The reason for this is because the degree centrality only shows a static topological structure and cannot represent the deeper understanding of subject matter and semantic level. However, the degree of outcome convergence proposed in this study is the only significant predictor $(t=3.002, p=0.005)$. Therefore, the degree of outcome convergence is an effective predictor that outperforms all the previous predictors.

\section{Discussion}

The present study aims to develop a precise, objective, and reusable method in order to assess collaborative learning processes and group performance through the lens of knowledge convergence. We explored the relationship between knowledge convergence and group performance. The results revealed that the number of activated common knowledge is related to the outcome of a collaborative learning process. The results also showed that the degree of outcome convergence could effectively predict group performance. Consistent with Kapur et al. (2008), the degree of outcome convergence was a powerful predictor for group performance. Our results also confirm Cannon-Bowers and Salas' earlier findings that knowledge 
convergence was regarded as a predictor for group performance (Cannon-Bowers and Salas 2001). Our results also corroborate the finding discovered by Fischer and Mandl (2005), who found that participants converged in knowledge learn more than those who did not. In addition, our findings shed light on the assessment of collaborative learning process quality. One of the significant contributions is that the interaction process can be visualized by the activating knowledge map at any timestamp. Another contribution of this study is that the visualized process convergent knowledge highlighted the focus of collaborative learning. The degree of process convergence can provide useful feedback for the best knowledge convergence, and the degree of outcome convergence can strongly predict group performance.

As a basic concept, knowledge convergence was emerged as a result of group interaction and reciprocal influence. Convergence on correct interpretations is a goal of collaborative learning (Hübscher-Younger and Narayanan 2003). Knowledge convergence can reflect the social interaction nature of knowledge co-construction. In this study, we have derived an operational definition and developed two formulas to quantitatively measure the level of knowledge convergence for collaborative learning. The definition of knowledge convergence in this study emphasizes the activated common knowledge during a collaborative learning process. It is different from the definition proposed by Jeong and Chi (2007) who conceptualized knowledge convergence as an increase in common knowledge. It is also distinguished from knowledge equivalence and shared knowledge as defined by Weinberger et al. (2007).

Furthermore, we have also provided a process-oriented measurement of knowledge convergence and demonstrated ways to measure the degree of process and outcome convergence. The advantage of this method is that it can measure the semantic relationships and structure of knowledge. Additionally, it can be used to examine the process of knowledge convergence by fully utilizing temporal information. Third, it is more precise than previous studies due to the use of the knowledge map approach. The initial knowledge map is viewed as the objective reference when coding information. This process-oriented methodology aims to analyze the process of knowledge convergence by computing the variation of quantity of activation. This method can also play an instrumental role in understanding the nature of knowledge convergence and in revealing novel insight into the process of convergence. Meanwhile, this method sheds light on how knowledge convergence evolves over time, and how the variation of evolution can explain group performance.

From the perspective of complex systems, knowledge convergence is an emergent behavior that arises from micro-level dynamics of interactions between group members. This emergent complex behavior is a macro-level phenomenon at the group level that cannot be attributed to any individual member in a group. Therefore, this study focuses on the process and degree of knowledge convergence at the group level. We argue that knowledge convergence is not suitable to be studied at the individual level, and it should be analyzed at the group level. Previous studies have, however, investigated the knowledge convergence phenomenon at the individual level (Jeong and Chi 2007). In fact, as Stahl (2011)proposed, group 
knowledge construction should be studied at the group level as only focusing on the individual cannot be used to systematically study and understand the nature of knowledge convergence.

We summarized some conditions of occurrence of convergence by analyzing thirty-one groups' discourse. Firstly, similar prior knowledge owned by group members may lead to the occurrence of knowledge convergence. Secondly, shared input is also one of the sources of knowledge convergence. Thirdly, when learners build and elaborate on the common knowledge, knowledge convergence can occur because the quantity of activation will become increasingly higher. Also, the negotiation of the shared understanding may lead to knowledge convergence. Finally, knowledge convergence can be more effective when group members share knowledge with the objective of reaching a common goal.

Collaborative learning is characterized by the spiral of reciprocity (Salomon and Perkins 1998). While it was often assumed that convergent processes were effective during collaborative learning, it was also recognized that divergent processes were beneficial (Ickes and Gonzalez 1996; Puntambekar 2006). Divergent ideas have a significant effect on collaborative interactions (Hoadley and Enyedy 1999; Stahl 2002; Jorczak 2011). In fact, during the early stages of collaborative learning, divergence is encouraged to represent all aspects of a subject matter. We believe that divergence is also an important means of reaching convergence. Divergence refers to the alternative or different views regarding the same topic. The generation of divergence can help to achieve convergence by jointly solving the conflict, thus deepening the understanding of the subject matter. Accordingly, teachers should encourage participants to diverge before converging. From divergence to convergence in collaborative learning is the most ideal situation. Generally speaking, before collaboration, the degree of divergence is higher than the degree of convergence. During collaboration, however, the extent of convergence will increase spirally. After collaboration, group members will become more convergent with respect to common understanding and knowledge. Therefore, knowledge convergence is a gradual, evolutionary, and spiral process.

From a practical standpoint, knowledge convergence research can promote an understanding of the characteristics of effective interactions, which, in turn, lead to improved group performance. Knowledge convergence has the potential to identify high-performing groups and to diagnose which group is having problem. Meanwhile, instructional supports play an increasingly important role in facilitating knowledge convergence as it is difficult to achieve knowledge convergence without additional support during a collaborative learning process. External representation tools, teachers' guidance, and knowledge awareness tools can serve as vehicles for achieving knowledge convergence by providing convergence-related external representations. Similarly, collaboration scripts are regarded as instructional support for knowledge convergence (Fischer and Mandl 2005). Accordingly, teachers can promote group's knowledge convergence by applying these strategies to attain instructionally desirable levels. 


\section{Conclusions}

Knowledge convergence has become a central issue in the field of collaborative learning. Understanding how knowledge convergence occurs during a collaborative learning process can provide valuable suggestions for making interactions more successful. In this paper, we have developed quantitative methods of knowledge convergence as a great supplement to qualitative analysis. A process-oriented methodology was proposed to quantitatively assess the quality of a collaborative learning process and group performance. The results show that the degree of process convergence and the number of activated common knowledge can quantify the quality of a collaborative learning process. The degree of outcome convergence can effectively predict group performance. The indicator developed in this study has a stronger predictive power than the other commonly used predictors. This study validates that knowledge convergence is a useful approach to assess the quality of collaborative learning process and outcome.

There are several limitations in this study. First, the measurement for knowledge convergence is limited to explicit knowledge because it is difficult to assess the level of convergence of implicit knowledge. Second, the reliability of coding information flows needs to be improved in the future. In this study, there are only two coders to rate the information produced from the collaborative learning process. Third, this study was conducted in a face-to-face collaborative learning environment. Whether the conclusion can be generalized to the computer-supported collaborative learning context needs a further study.

Future research should address how to assess the degree of implicit knowledge convergence. Meanwhile, further studies are needed to examine the generalization of conclusions in synchronous and asynchronous communication modes and explore how to automatically provide just-in-time feedback according to the degree of process convergence. Finally, the occurrence of knowledge convergence requires the support of external representation tools and teachers' guidance. To better achieve knowledge convergence, how teachers facilitate and scaffold interactions should be concerned and explored thoroughly for collaborative learning.

Acknowledgments This study was supported by the Twelfth Five-Year Plan of the National Educational Science in China (ECA110330).

\section{References}

Aviv, R., Erlich, Z., Ravid, G., \& Geva, A. (2003). Network analysis of knowledge construction in asynchronous learning networks. Journal of Asynchronous Learning Networks, 7(3), 1-23.

Cannon-Bowers, J. A., \& Salas, E. (2001). Reflections on shared cognition. Journal of Organizational Behavior, 22(2), 195-202.

Clariana, R., Draper, D. \& Land, S. (2011). An automated measure of group knowledge structure convergence. Paper presented at the annual meeting of the AECT International Convention. Retrieved October 7, 2012, from http://www.personal.psu.edu/rbc4/AECT_2011.pdf.

De Laat, M., Lally, V., Lipponen, L., \& Simons, R. J. (2007). Investigating patterns of interaction in networked learning and computer-supported collaborative learning: A role for Social Network Analysis. International Journal of Computer-Supported Collaborative Learning, 2(1), 87-103. 
Fahy, P. J., Crawford, G., \& Ally, M. (2001). Patterns of interaction in a computer conference transcript. The International Review of Research in Open and Distance Learning, 2(1), 1-24.

Fischer, F., \& Mandl, H. (2005). Knowledge convergence in computer-supported collaborative learning: The role of external representation tools. The Journal of the Learning Sciences, 14(3), 405-441.

Gress, C. L., Fior, M., Hadwin, A. F., \& Winne, P. H. (2010). Measurement and assessment in computersupported collaborative learning. Computers in Human Behavior, 26(5), 806-814.

Hoadley, C. M., \& Enyedy, N. (1999). Between information and communication: middle spaces in computer media for learning. In C. M. Hoadley, \& J. Roschelle (Eds.), CSCL_99 Proceedings of computer support for collaborative learning (pp. 242-251). Mahwah, NJ: Lawrence Erlbaum Associates.

Hübscher-Younger, T., \& Narayanan, N. H. (2003). Authority and convergence in collaborative learning. Computers and Education, 41(4), 313-334.

Ickes, W., \& Gonzalez, R. (1996). "Social" cognition and social cognition: From subjective to the intersubjective. In J. L. Nye \& A. M. Brower (Eds.), What's social about social cognition? (pp. 285-309). Thousand Oaks: Sage.

Jarmon, L., Traphagan, T., Mayrath, M., \& Trivedi, A. (2009). Virtual world teaching, experiential learning, and assessment: An interdisciplinary communication course in Second Life. Computers and Education, 53(1), 169-182.

Jeong, H., \& Chi, M. (2007). Knowledge convergence and collaborative learning. Instructional Science, 35(4), 287-315.

Jorczak, R. L. (2011). An information processing perspective on divergence and convergence in collaborative learning. International Journal of Computer-Supported Collaborative Learning, 6(2), 207-221.

Kapur, M., Voiklis, J., \& Kinzer, C. K. (2008). Sensitivities to early exchange in synchronous computersupported collaborative learning (CSCL) groups. Computers and Education, 51(1), 54-66.

Kapur, M., Voiklis, J., \& Kinzer, C. K. (2011). A complexity-grounded model for the emergence of convergence in CSCL groups. In S. Puntambekar, G. Erkens, \& C. Hmelo-Silver (Eds.), Analyzing interactions in CSCL: Methods, approaches and issues (pp. 3-23). New York: Springer.

Klimoski, R., \& Mohammed, S. (1994). Team mental model: Construct or metaphor. Journal of Management, 20(2), 403-437.

Li, X., Chu, K., Ki, W. W., \& Woo, M. M. (2012). Using a wiki-based collaborative process writing pedagogy to facilitate collaborative writing among Chinese primary school students. Australasian Journal of Educational Technology, 28(1), 159-181.

Macdonald, J. (2003). Assessing online collaborative learning: Process and product. Computers and Education, 40(4), 377-391.

Meier, A., Spada, H., \& Rummel, N. (2007). A rating scheme for assessing the quality of computersupported collaboration processes. International Journal of Computer-Supported Collaborative Learning, 2(1), 63-86.

Nandi, D., Hamilton, M., Chang, S., \& Balbo, S. (2012). Evaluating quality in online asynchronous interactions between students and discussion facilitators. Australasian Journal of Educational Technology, 28(4), 684-702.

Prokofieva, M. (2013). Evaluating types of students' interactions in a wiki-based collaborative learning project. Australasian Journal of Educational Technology, 29(4), 496-512.

Puntambekar, S. (2006). Analyzing collaborative interactions: Divergence, shared understanding and construction of knowledge. Computers and Education, 47(3), 332-351.

Roschelle, J. (1996). Learning by collaborating: Convergent conceptual change. In T. Koschmann (Ed.), CSCL: Theory and practice of an emerging paradigm (pp. 209-248). Mahwah, NJ: LawrenceErlbaum Associates Inc.

Roschelle, J., \& Teasley, S. D. (1995). The construction of shared knowledge in collaborative problem solving. In C. E. O’Malley (Ed.), Computer-supported collaborative learning (pp. 69-97). Berlin: Springer-Verlag.

Salomon, G., \& Perkins, D. N. (1998). Individual and social aspects of learning. Review of Research in Education, 23(1), 1-24.

Spemann, K., \& Fischer, F. (2011). Quantifying qualities in collaborative knowledge construction: The analysis of online discussions. In S. Puntambekar, G. Erkens, \& C. Hmelo-Silver (Eds.), Analyzing interactions in CSCL: Methods, approaches and issues (pp. 247-268). New York: Springer.

Stahl, G. (2002). Rediscovering CSCL. In R. H. T. Koschmann \& N. Miyake (Eds.), CSCL 2: Carrying forward the conversation (pp. 275-296). Mahwah, NJ: Erlbaum. 
Stahl, G. (2011). How to study group cognition. In S. Puntambekar, G. Erkens, \& C. Hmelo-Silver (Eds.), Analyzing interactions in CSCL: Methods, approaches and issues (pp. 107-130). New York: Springer.

Stahl, G., Koschmann, T., \& Suthers, D. (2006). Computer supported collaborative learning: An historical perspective. In R. K. Sawyer (Ed.), Cambridge handbook of the learning sciences (pp. 409-426). New York: Cambridge University Press.

Strijbos, J. W. (2011). Assessment of (computer-supported) collaborative learning. Learning Technologies. IEEE Transactions on Learning Technologies, 4(1), 59-73.

Strijbos, J., \& Fischer, F. (2007). Methodological challenges for collaborative learning research. Learning and Instruction, 17(4), 389-393.

van Aalst, J., \& Chan, C. K. (2007). Student-directed assessment of knowledge building using electronic portfolios. The Journal of the Learning Sciences, 16(2), 175-220.

Weinberger, A., \& Fischer, F. (2006). A framework to analyze argumentative knowledge construction in computer-supported collaborative learning. Computers and Education, 46(1), 71-95.

Weinberger, A., Stegmann, K., \& Fischer, F. (2007). Knowledge convergence in collaborative learning: Concepts and assessment. Learning and Instruction, 17(4), 416-426.

Zemel, A., Xhafa, F., \& Stahl, G. (2005). Analyzing the organization of collaborative math problemsolving in online chats using statistics and conversation analysis. In H. Fukś, S. Lukosch, \& A. C. Salgado (Eds.), Groupware: Design, implementation, and use (pp. 271-283). Berlin: Springer.

Zhang, J., Scardamalia, M., Reeve, R., \& Messina, R. (2009). Designs for collective cognitive responsibility in knowledge-building communities. The Journal of the Learning Sciences, 18(1), 7-44.

Zheng, L., Yang, K., \& Huang, R. (2012). Analyzing interactions by an IIS-Map-Based method in faceto-face collaborative learning: An empirical study. Educational Technology and Society, 15(3), $116-132$.

Zottmann, J. M., Stegmann, K., Strijbos, J. W., Vogel, F., Wecker, C., \& Fischer, F. (2013). Computersupported collaborative learning with digital video cases in teacher education: The impact of teaching experience on knowledge convergence. Computers in Human Behavior, 29(5), 2100-2108.

Lanqin Zheng currently works as an assistant professor and supervisor for postgraduate at the School of Educational Technology in Beijing Normal University. She received her PhD from Beijing Normal University, China. Currently, Dr. Lanqin Zheng is a postdoctoral fellow at Athabasca University, Canada. Her research interests include computer-supported collaborative learning, big data learning analytics, and instructional design.

Nian-Shing Chen is Chair Professor at the Department of Information Management in the National Sun Yat-sen University, Taiwan. He has published over 350 papers in the international referred journals, conferences, and book chapters. Prof. Chen received two outstanding research awards from the National Science Council, Taiwan in 2008 and 2011-2013. His current research interests include assessing e-Learning course performance; online synchronous teaching \& learning; mobile \& ubiquitous learning; Game-based learning; and Cognition \& Gesture-based Learning. Prof. Chen is serving as an editorial board member for many international journals and guest editors for special issues of international journals. He has also organized and chaired numerous international conferences and workshops in the area of advanced learning technologies. Professor Chen is a senior member of IEEE, ACM, and the Chair for the IEEE Technical Committee on Learning Technology (http://ttf.ieee.org/). He is Co-Editor-InChief of the SSCI indexed Journal of Educational Technology \& Society.

Ronghuai Huang received the PHD degree in 2000 in educational technology from Beijing Normal University. He is a professor of school of Educational Technology, Faculty of Education, Beijing Normal University. His research interests include smart learning environment, e-textbooks, and computersupported collaborative learning. He has been involved in many research projects and published many papers in educational technology journals.

Kaicheng Yang received the PHD degree in 1999 in educational technology from Beijing Normal University. He is a professor of school of Educational Technology, Faculty of Education, Beijing Normal University. His research interests include computer aided learning and instructional design. 